\title{
Solar flare detection sensitivity using the South America VLF Network (SAVNET)
}

\author{
Jean-Pierre Raulin, ${ }^{1}$ Fernando C. P. Bertoni, ${ }^{1}$ Hernan R. Gavilán, ${ }^{1}$ Walter Guevara-Day, ${ }^{2}$ \\ Rodolfo Rodriguez, ${ }^{3}$ Germán Fernandez, ${ }^{4}$ Emilia Correia, ${ }^{1,5}$ Pierre Kaufmann, ${ }^{1,6}$ \\ Alessandra Pacini, ${ }^{5}$ Tardelli R. C. Stekel, ${ }^{7}$ Washington L. C. Lima, ${ }^{8}$ Nelson J. Schuch, ${ }^{7}$ \\ Paulo R. Fagundes, ${ }^{9}$ and Rubens Hadano ${ }^{10}$
}

Received 3 December 2009; revised 15 January 2010; accepted 18 February 2010; published 7 July 2010.

[1] We present recent observations of Sudden Phase Anomalies due to subionospheric propagation anomalies produced by solar X-ray flares. We use the new South America VLF Network (SAVNET) to study 471 ionospheric events produced by solar flares during the period May 2006 to July 2009 which corresponds to the present minimum of solar activity. For this activity level, we find that $100 \%$ of the solar flares with a $\mathrm{X}$-ray peak flux above $5 \times 10^{-7} \mathrm{~W} / \mathrm{m}^{2}$ in the $0.1-0.8 \mathrm{~nm}$ wavelength range produce a significant ionospheric disturbance, while the minimum $\mathrm{X}$-ray flux needed to do so is about $2.7 \times 10^{-7} \mathrm{~W} / \mathrm{m}^{2}$. We find that this latter minimum threshold is dependent on the solar cycle, increasing when the Sun is more active, thus confirming that the low ionosphere is more sensitive during periods of low solar activity. Also, our findings are in agreement with the idea that the ionospheric D-region is formed and maintained by the solar Lyman- $\alpha$ radiation outside solar flare periods.

Citation: Raulin, J.-P., et al. (2010), Solar flare detection sensitivity using the South America VLF Network (SAVNET), J. Geophys. Res., 115, A07301, doi:10.1029/2009JA015154.

\section{Introduction}

[2] The monitoring and analysis of very low frequency (VLF; 3-30 kHz) propagation anomalies is a powerful tool to study the low altitude Earth's ionosphere like the diurnal $\mathrm{C}$ and $\mathrm{D}$ regions at an altitude range of $60-70 \mathrm{~km}$. This portion of the ionosphere is not accessible to stratospheric balloons and is well below most satellites orbits. VLF waves propagate over long distances within the Earth-ionosphere waveguide (EIW), and their characteristics in phase $\Phi$ and amplitude $A$ inform about the electrical properties of the waveguide's conducting boundaries (i.e., the surface of the

\footnotetext{
${ }^{1}$ Centro de Radio Astronomia e Astrofísica Mackenzie (CRAAM), Universidade Presbiteriana Mackenzie, São Paulo, Brazil.

${ }^{2}$ Comisión Nacional de Investigación y Desarrollo Espacial (CONIDA), Lima, Peru.

${ }^{3}$ Universidad de Piura (UDEP), Piura, Peru.

${ }^{4}$ Complejo Astronómico El Leoncito (CASLEO), San Juan, Argentina.

${ }^{5}$ Instituto Nacional de Pesquisas Espaciais (INPE), São José dos Campos, Brazil.

${ }^{6}$ Centro de Componentes Semicondutores, Universidade Estadual de Campinas (UNICAMP), Campinas, Brazil.

${ }^{7}$ Centro Regional Sul-INPE (CRS/INPE), Universidade Federal de Santa Maria (UFSM), Santa Maria, Brazil.

${ }^{8}$ Universidade Luterana do Brasil (ULBRA), Palmas, Brazil.

${ }^{9}$ Universidade do Vale do Paraíba (UNIVAP), São José dos Campos, Brazil.

${ }^{10}$ Radio Observatório do Itapetinga (ROI)-INPE/Mackenzie, Atibaia, Brazil.

Copyright 2010 by the American Geophysical Union. 0148-0227/10/2009JA015154
}

Earth and the low ionosphere plasma) [Wait and Spies, 1964].

[3] The main cause for the presence of the daytime ionospheric D-region is the solar Lyman- $\alpha(1216 \AA)$ radiation [Nicolet and Aikin, 1960] which progressively increases the electrical conductivity in the $70-90 \mathrm{~km}$ altitude range by ionizing nitric oxide (NO) molecules as the Sun rises. As a result, a relatively stable reflecting layer is formed at about $70 \mathrm{~km}$ which lasts under solar daytime conditions. At night, electron recombination processes overcome the ionization, and the $\mathrm{D}$ region disappears when the reflecting layer moves to about $90 \mathrm{~km}$ and is now formed by the bottom of the nighttime E region. Therefore, it is useful to study the properties of the low ionosphere by the mean of parameters which characterize the electrical conductivity (height) profile: the reference height $H^{\prime}$ (in kilometers) and the conductivity gradient $\beta$ (in kilometers ${ }^{-1}$ ) [Wait, 1959; Wait and Spies, 1964]. The reference height $H^{\prime}$ is the altitude where the electrical conductivity is constant at $2.5 \times 10^{5} \mathrm{~s}^{-1}$ and the conductivity gradient (or sharpness) informs how fast the conductivity changes with height.

[4] Departure from the above described quiescent situation occurs when there are ionization excesses in the low ionosphere. The reasons for that can be external sources of radiation like X-ray solar flares [Bracewell and Straker, 1949; Kaufmann and Paes de Barros, 1969; Muraoka et al., 1977], X-ray bursts from remote objects like magnetar bursts [Kaufmann et al., 1973; Rizzo Piazza et al., 1983; Tanaka et al., 2008; Raulin et al., 2009a], or meteor showers [Chilton, 


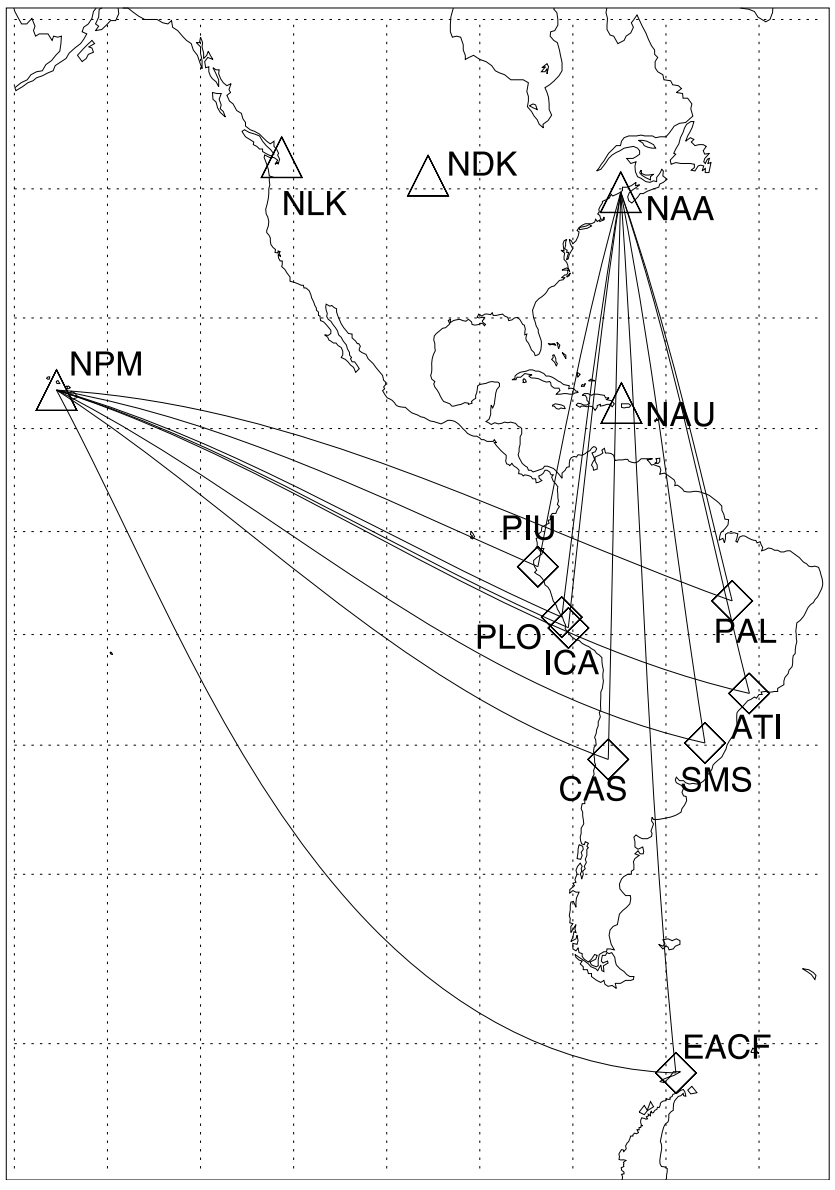

Figure 1. Examples of VLF propagation paths from transmitters NPM and NAA, to the receiver bases of the SAVNET array in Brazil, Peru, Argentina, and the Antarctic continent.

1961; Kaufmann et al., 1989]. The electrical properties of the EIW can also change due to perturbations from the inside of the waveguide like atmospheric lightning causing precipitation of initially trapped electrons [Cummer, 1997], Transient Luminous Effects (TLEs) like red sprites and elves [Inan et al., 1995; Pasko et al., 1995], and natural phenomena related to seismic-electromagnetic effects [Hayakawa et al., 1996].

[5] During solar flares when the solar active regions plasma is heated up to few tens of millions of degrees, increases of a few orders of magnitude of the solar X-ray emission are observed at the Earth orbit. The photons with $\lambda \leq 2 \AA$ can reach altitudes below the reference height $H^{\prime}$ [Pacini and Raulin, 2006], producing significant ionization enhancements in the low ionosphere which results in variations of one or both of the Wait parameters. Lowering of $H^{\prime}$ is observed in the phase of long-distance propagating waves as Sudden Phase Anomalies (SPA), that is, as advances of the phase of the transmitted waves. The SPA intensity $\Delta \Phi$ depends on the X-ray flux $\left(P_{x}\right)$ spectrum, and time of occurrence of the solar event as well as on the characteristics of the VLF propagation path. From this relation, it is generally possible to infer the lower soft X-ray flux $\left(P_{x m}\right)$ needed to produce a ionospheric event, and $P_{x m}$ can be further used to deduce the electron density enhancement in the D-region at the time of solar flares and/or to improve the determination of recombination coefficients which are generally poorly known. For space weather studies, and in particular for the present study, it is important to know whether $P_{x m}$ depends on the level of solar activity, and thus confirm that the low ionosphere sensitivity changes with solar activity conditions [Pacini and Raulin, 2006].

[6] In this paper we study the relation between solar X-ray flares and the subsequent ionospheric disturbances detected by a new VLF instrumental facility, the South America VLF Network (SAVNET). SAVNET is an international project between Brazil, Peru, and Argentina dedicated to monitor the effects of the solar activity in the low ionosphere and in particular over the South Atlantic Magnetic Anomaly (SAMA) region. In the next section we briefly present the instrumentation used and describe the methodology adopted as well as the observational results we have obtained. We then discuss these results before presenting our concluding remarks.

\section{Instrumentation, Data Analysis, and Observational Results}

[7] The SAVNET installation has been performed in the 2007-2009 time period, and the array is currently composed of eight VLF tracking stations located in Brazil, Peru, and Argentina. In Figure 1 we show the location of the SAVNET receivers bases as well as the positions of the transmitters. Examples of VLF propagation paths, part of Great Circle Paths (GCP), are shown from the transmitters NAA and NPM. Each receiver base is composed of three electromagnetic sensors, and the VLF signals received from the powerful transmitters are amplified and then digitized using a commercial audio card. The crystal of the audio card provides a clock signal which is locked to a GPS internal clock signal (1 PPS), and this ensemble provides a phase determination of the incoming wave without any drift. The resulting phase signal presents a precision (root mean square, RMS) of about $0.05-0.07 \mu \mathrm{s}$, which corresponds to about less than $1^{\circ}$ depending on the frequency of the incoming VLF wave. In this paper we use the database from the 13,060 km long VLF propagation path between the transmitter NPM (Hawaii, $21.4 \mathrm{kHz}$ ) and the receiver ATI (Atibaia, SP, Brazil), path which is hereafter mentioned as NPM-ATI. For more details on the SAVNET instrumental setup and scientific goals, see Raulin et al. [2009b, 2009c].

[8] The solar flare database used in this paper is composed of soft X-ray fluxes from GOES detectors which record the solar radiation in two photon energy channels, 0.1-0.8 and 0.05-0.4 nm (NOAA, Space Weather Prediction Center). The fluxes detected by GOES instruments mainly come from the thermal free-free emission produced in hot solar active regions, where the plasma temperature lies in the range 1-3 MK. During solar flares, soft X-ray fluxes are significantly enhanced by several orders of magnitude due to an increase of the plasma temperature or the plasma emission measure or both.

[9] Since May 2006, 471 flares have been detected and ordered using the GOES classification (GOES class). This classification is based on the peak power $P_{x}$ (watts per 


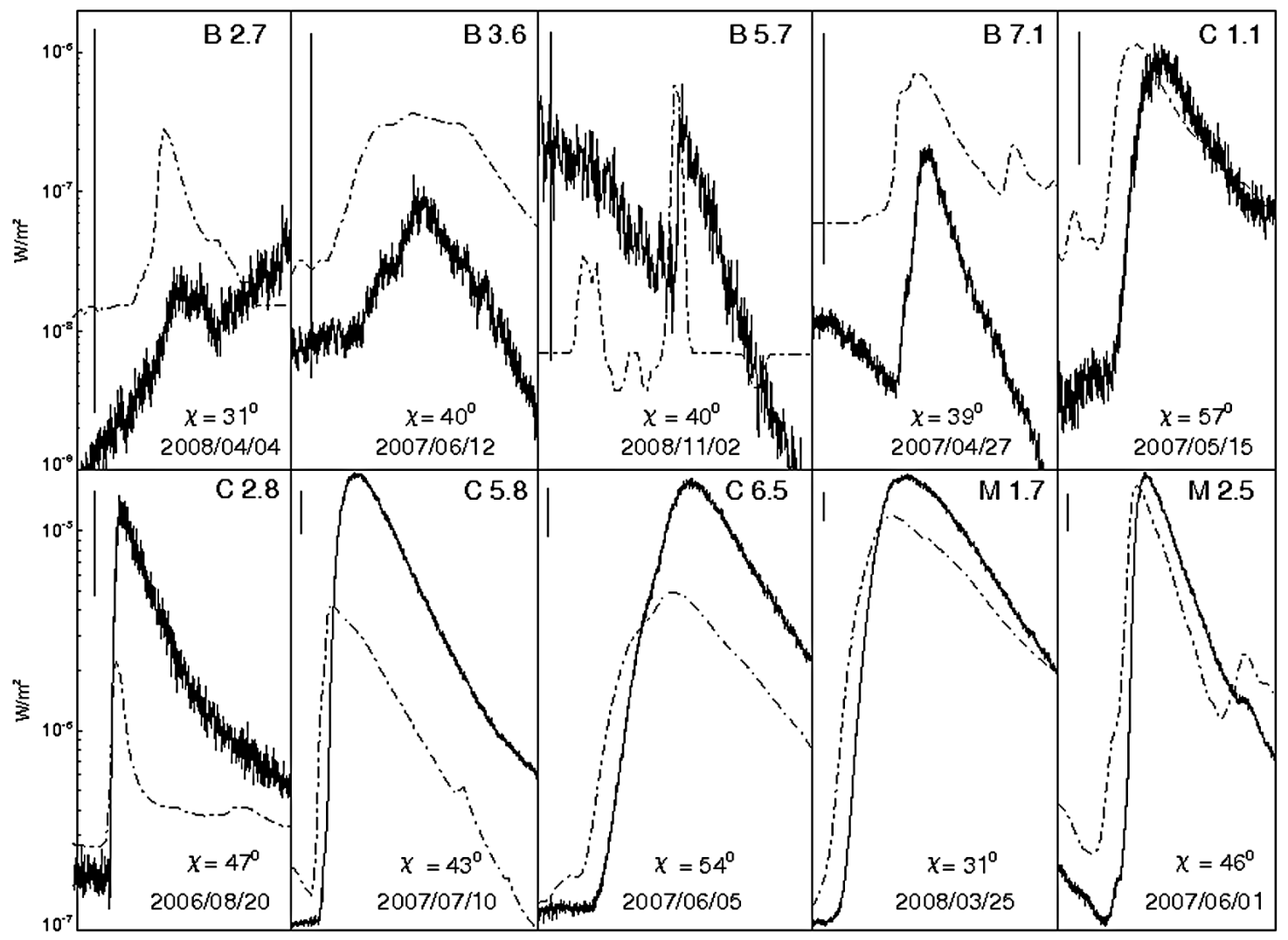

Figure 2. Examples of solar flares detected by the SAVNET array using the VLF propagation path NPM-ATI. One hour time duration soft X-ray fluxes (dash-dot) are compared to the phase excesses ( 3 s time integrated noisy full line). The date and the GOES class of the event are indicated as well as the mean solar zenith angle along the propagation path at the time of each solar event. The vertical thick lines represent a phase excess of $15^{\circ}$. Note the very small solar flare in the upper left corner. Such an event does represent the power detection lower limit.

meter squared) detected in the $0.1-0.8 \mathrm{~nm}$ channel, using the following rule and ranges: $\mathrm{B}$, class for $P_{x}$ in the range $10^{-7}$ to $10^{-6} \mathrm{~W} / \mathrm{m}^{2} ; \mathrm{C}$, class for $P_{x}$ in the range $10^{-6}$ to $10^{-5} \mathrm{~W} / \mathrm{m}^{2} ; \mathrm{M}$, class for $P_{x}$ in the range $10^{-5}$ to $10^{-4} \mathrm{~W} / \mathrm{m}^{2}$; $\mathrm{X}$, class for $P_{x}$ greater than $10^{-4} \mathrm{~W} / \mathrm{m}^{2}$. Thus, a C 1.5 GOES class solar flare does present a peak flux of $1.5 \times 10^{-6} \mathrm{~W} / \mathrm{m}^{2}$ in the $0.1-0.8 \mathrm{~nm}$ channel. From the original solar flare database, we removed those solar events for which the mean solar zenithal angle, estimated along the whole VLF propagation path was greater than $90^{\circ}$. For the east-west oriented propagation path (NPM-ATI) used in this work, we also did not take into account the solar flares which occurred right at, or close to, the time of VLF modal minima, since it may mask the phase advance observed by the VLF receiver. The criterion we have adopted for flare detection using the $1 \mathrm{~s}$ time constant VLF phase signal corresponds to an increase of about $1.5 \sigma$ (RMS) compared to the mean preflare phase.

[10] As a result we present in Figure 2 one-hour sample cases of ten X-ray flares of different classes from $\mathrm{B}$ to $\mathrm{M}$ GOES class. The dash-dot lines show the $0.1-0.8 \mathrm{~nm}$ soft $\mathrm{X}$-ray flux time profiles which are compared to the VLF phase records (full line) observed simultaneously. In each plot we indicate the mean solar zenith angle, $\chi$, estimated along the whole path at the time of the peak of the solar event. It has been computed by dividing each VLF propagation path in 100 parts and calculating for each piece a solar zenithal angle $\chi_{i}(i=1,100)$. The $\chi$ is then calculated as the mean value of the $\chi_{i}$ angles. The thick vertical lines in the left part of each panel represent a phase excess of $15^{\circ}$. Therefore, Figure 2 gives an illustration of how the very low frequency receivers from the SAVNET array observe solar flares of different X-ray flux. Note in particular in the upper left corner the SPA associated with a small B 2.7 solar event, for which the phase advance was $2.5^{\circ}$ which corresponds to a phase advance of $0.3 \mu \mathrm{s}$ at the frequency of $21.4 \mathrm{kHz}$. Such a solar event is thus detected as an increase of the phase of about $4 \sigma$.

[11] The main result of this paper is shown in Figure 3 and is related to the capability of detecting solar flare events using a VLF technique. We can define this ability as the probability $P$ that a given flare of X-ray power $P_{x}$ produces a sudden phase anomaly (SPA). $P$ is then obtained by the ratio of the number of solar events detected in a given class to the total number of solar events which occurred in the same class. The full line histogram shows the probability of detecting solar flares with $\chi<40^{\circ}$ as a function of $P_{x}$, and the dashed histogram shows the same for flares with 


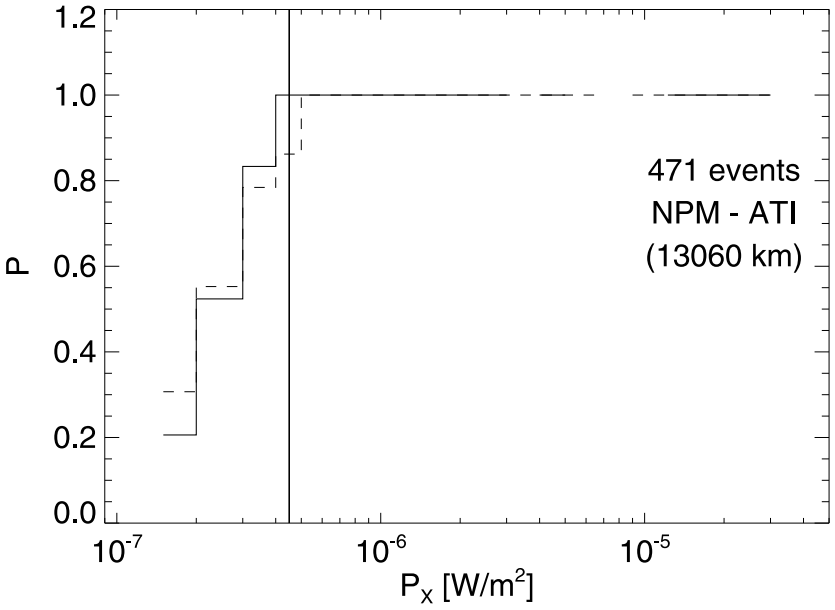

Figure 3. Solar flare probability detection $P$, as a function of the soft X-ray peak flux $P_{x}$ for the long NPM-ATI VLF propagation path, and for solar zenith angle lower (dashed line) than $70^{\circ}$ and lower (full line) than $40^{\circ}$.

$\chi<70^{\circ}$. The vertical thick bar indicates the value of $P_{x}$ for which the probability $P$ becomes $100 \%$. Thus, our results indicate that solar flares with a peak X-ray flux $\geq 5 \times$ $10^{-7} \mathrm{~W} / \mathrm{m}^{2}$ (GOES B5 class or higher) will be detected with a probability of $100 \%$ in the low ionosphere.

[12] The lower detection limit, $P_{x m}$, which would correspond to the lower soft $\mathrm{X}$-ray power needed to produce a SPA is about $2.7 \times 10^{-7} \mathrm{~W} / \mathrm{m}^{2}$ corresponding to a GOES class for which solar events are detected with a probability of $50 \%$. A typical time profile of such an event is shown in Figure 2 (upper left panels).

\section{Discussion}

[13] In the previous section we showed the capability of the SAVNET instrumental facility to detect solar flares, even small solar events of GOES class B. We now compare our findings with related earlier works.

[14] A direct comparison of our results can be performed with those obtained by Comarmond [1977]. The author studied about 520 solar flare events during a period of high to medium solar activity levels between December 1968 and January 1971 using the $6970 \mathrm{~km}$ long east-west oriented VLF propagation path NWC (Australia)-TANANARIVE (Madagascar). When only the solar flares with $\chi<40^{\circ}$ were taken into account, a $75 \%$ detection probability is achieved for $P_{x}$ in the range $5.6 \times 10^{-6}$ to $1.0 \times 10^{-5} \mathrm{~W} / \mathrm{m}^{2}$ (C 5.6-M 1.0 GOES classes), that is about one order of magnitude higher than our results shown in Figure 3. The detection probability for smaller flares in the range C 3.2-C 5.6 was about $25 \%$. As we will discuss below, the reason for this difference is due to the level of solar activity at that time.

[15] Most of the papers studying the relation between solar flares and the resulting response of the low ionosphere using the VLF technique deal with the correlation between the $\mathrm{X}$-ray peak power in a given photon energy range $\left(P_{x}\right)$ and the phase $(\Delta \Phi)$ and/or amplitude $(\Delta A)$ changes which are subsequently observed [Kaufmann and
Paes de Barros, 1969; Comarmond, 1977; Muraoka et al., 1977; Pant, 1993; Pant et al., 1983; Kaufmann et al., 2002; McRae and Thomson, 2004; Thomson et al., 2005; Pacini, 2006; Raulin et al., 2006; Zigman et al., 2007]. In general, the $P_{x}$ versus $\Delta \Phi$ (or $\Delta A$ ) plots do present a good correlation for both variables, and it is possible to identify the faintest solar events detected by extrapolating the correlation toward these small events deducing a minimum soft $\mathrm{X}$-ray flux, $P_{x m}$. This procedure does not inform on the probability of such events to produce an ionization excess in the low ionosphere; however, it tells us that they were actually detected using the VLF tracking technique.

[16] The result of the previously explained procedure is summarized in the Table 1 where we show the reference works, the VLF propagation path used and its length ( $L$ in megameters) and the frequency of observation (in kilohertz). The values obtained for $P_{x m}$ and the corresponding classes are also indicated. Table 1 also shows the epoch of occurrence of the solar events studied, and the level of solar activity at that time. This level has been estimated from the composite Lyman- $\alpha$ time profile from 1947 to the actual epoch [Woods et al., 2000] combining measurements and modeling results, being from 2003 to the present time actual measurements from TIMED/SEE and SORCE space missions [Woods et al., 2005; Rottman et al., 2006]. To obtain the mean value indicated in Table 1 (third column from left) we have preformed an average of the Lyman- $\alpha$ photon flux over the period of the solar flare observations.

[17] Our results on the probability $P$ are not strongly dependent on the solar zenith angle $\chi$. This is illustrated in Figure 3 for solar flares for which $\chi<70^{\circ}$ and for solar flares for which $\chi<40^{\circ}$. This is in agreement also with previous works which found no dependency with $\chi$, for $\chi$ values below $60^{\circ}-70^{\circ}$ [Kaufmann et al., 2002; Pant et al., 1983].

[18] A clear indication from Table 1 is that the minimum soft X-ray flux needed to produce a phase change during a solar flare is increasing as the level of solar activity is higher. We illustrate this property in Figure 4 where we have plotted $P_{x m}$ as a function of the mean level of solar Lyman- $\alpha$ radiation using the referenced works and corresponding numbers in Table 1 . We can see a very good correlation which emphasizes the fact that the low ionospheric response to solar photons is solar cycle dependent, being more sensitive at times of low solar activity. Such property was already shown in the work of Raulin et al. [2006] and Pacini and Raulin [2006]. Similarly, McRae and Thomson [2000, 2004] found that the ionospheric reference height $H^{\prime}$ was higher during solar activity minima, a fact that can also be interpreted as different ionospheric sensitivity for different solar activity levels. The correlation shown in Figure 4 clearly suggests that for a solar flare to be detected in the low ionosphere, the corresponding ionization should overcome that due to the quiescent solar Lyman- $\alpha$ radiation. At the same time, this result is in complete agreement with the hypothesis that the solar Lyman- $\alpha$ radiation forms and maintains the undisturbed D-region [Nicolet and Aikin, 1960].

[19] Finally, we would like to mention the work of Muraoka et al. [1977] who found a lower X-ray threshold, $P_{x m}$ of $1.5 \times 10^{-6} \mathrm{~W} / \mathrm{m}^{2}$ (C 1.5 GOES class) when studying the SPAs associated with $\sim 45$ solar flares in the period July 
1974-June 1975. For this period, the Lyman- $\alpha$ composite data show a mean photon flux of the order of $4 \times 10^{11} \mathrm{ph}$. $\mathrm{cm}^{-2} \cdot \mathrm{s}^{-1}$ such that the corresponding point $[4 ; 0.18]$ if displayed in Figure 4, will appear well above the correlation line. A reason for that may be related to the propagation path which was a high latitude $\left(>40^{\circ} \mathrm{N}\right)$, east-west oriented path between the transmitter NLK and the receiver HCM located in Japan. For about half of the year between October and March, the average (over the path length) solar zenith angle is $>40^{\circ}$, and it is greater than $60^{\circ}$ for the winter months between November and January. We also note that in this study the authors corrected the phase data using the minimum zenith angle over the VLF path rather than its mean value. In this case, one certainly underestimates the phase changes $\Delta \Phi$ and therefore may overestimate the value of $P_{x m}$ deduced from the $\Delta \Phi$ versus $P_{x}$ correlation plot. Another reason may be related to the fact that no soft X-ray data were available for the period studied by the authors, and $P_{x}$ values were therefore indirectly derived from $F_{\min }$ (i.e., the lowest frequency showing vertical ionospheric reflection which was measured close to the location of the VLF receiver [Sato, 1975]).

\section{Concluding Remarks}

[20] In this paper we have presented VLF subionospheric propagation anomalies associated with the occurrence of solar flares, using a new instrumental facility, SAVNET. In particular we concentrated on the capability of the new instrument in detecting solar events during the period corresponding to the present minimum of solar activity (20062009).

[21] The results have shown that solar flares with an $\mathrm{X}$-ray peak flux above $5 \times 10^{-7} \mathrm{~W} / \mathrm{m}^{2}$ (B 5 class) in the $0.1-0.8 \mathrm{~nm}$ energy range are detected with a probability of $100 \%$, and that the lower detection threshold is around $2.7 \times 10^{-7} \mathrm{~W} / \mathrm{m}^{2}$. Combining our results with earlier studies obtained for different solar activity levels, we find that the lower X-ray detection limit is an increasing function of the solar activity as characterized by the mean solar Lyman- $\alpha$ photon flux. These results are coherent with the idea that the quiescent diurnal low ionosphere at $\sim 70 \mathrm{~km}$ is maintained by the solar Lyman- $\alpha$ radiation [Nicolet and Aikin, 1960], and that it is more sensitive during period of low solar activity.

[22] Finally, we note that the high sensitivity of the VLF diagnostic to detect perturbation in the low ionosphere caused by solar flares, even small solar flares, may serve to stimulate the search for signatures from other objects. As we mentioned in the introduction of the paper, the SAVNET instrument has already detected perturbations caused by Anomalous X-ray Pulsars (or magnetars). An earlier and famous example was the flare of the Soft Gamma Repeater (SGR 1806-1820) which occurred in 27 December 2004 and which was widely described in the literature [Terasawa et al., 2005]. The interest in studying such objects lies in the fact that the energy output during a given burst can exceed by 10 to 15 orders of magnitude that released during the largest solar flares. Therefore, a better description of the physical processes leading to this huge liberation of energy depends on the derivation of good X-ray and $\gamma$-ray spectra. The VLF technique can thus provide a 


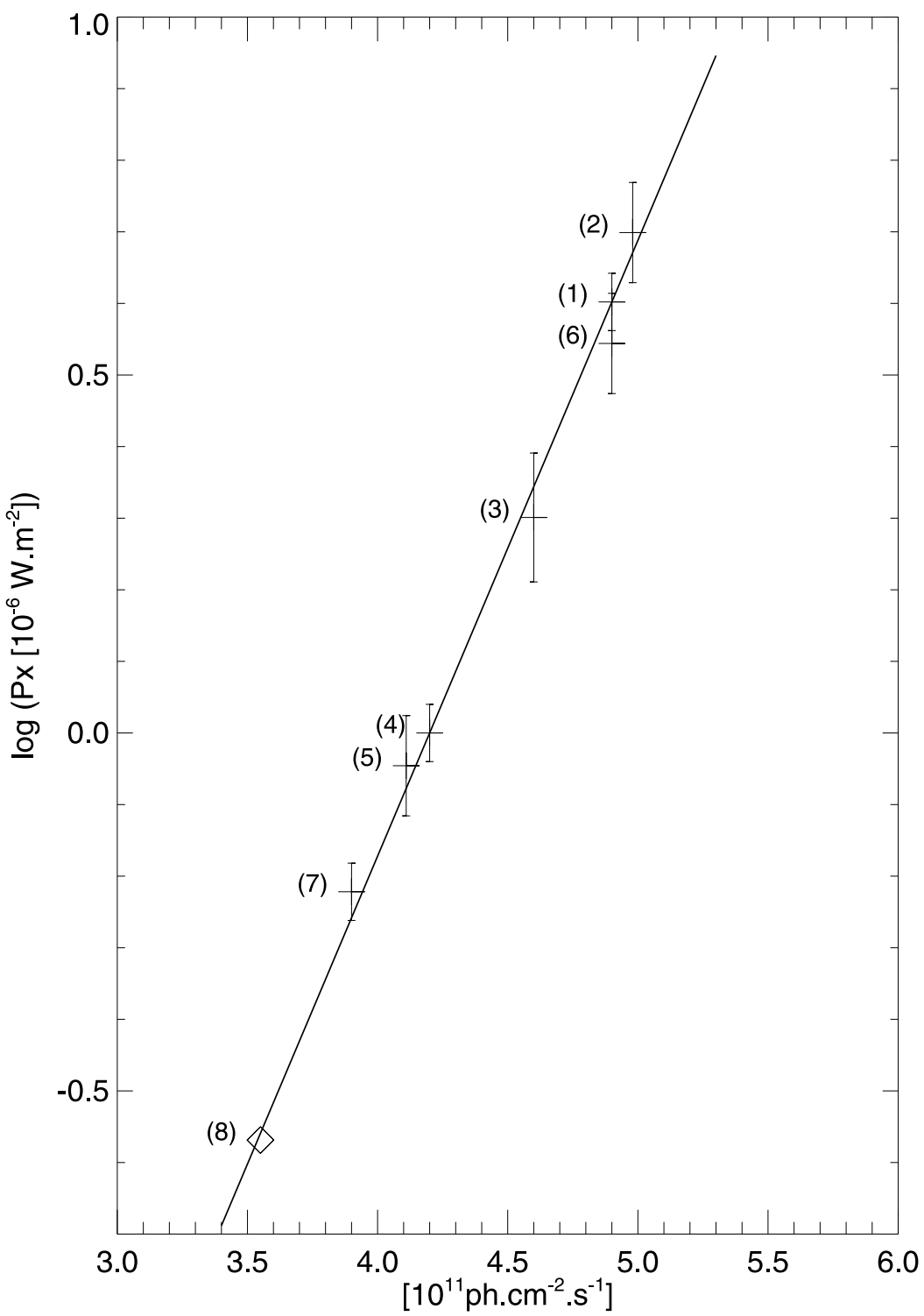

Figure 4. Correlation $\log \left[P_{x}\right]$ as a function of the mean Lyman- $\alpha$ solar flux. Numbers refer to the reference works listed in the right column of Table 1.

way of better determining the low energy part of spectra of celestial $\gamma$-ray flares, if they are intense enough to produce ionospheric perturbations.

[23] Acknowledgments. The authors thank two anonymous referees for comments and suggestions which have improved the quality of this paper. JPR would like to thank funding agencies MACKPESQUISA and CNPq (Proc. 304433/2004-7). FCPB thanks FAPESP (Proc. 2007/ 05630-1). The SAVNET project is funded by FAPESP (Proc. 06/02979) and received partial funds from $\mathrm{CNPq}(490124 / 2006-2)$ and Centre National de la Recherche Scientifique (CNRS). SAVNET has been participating to the International Heliophysical Year program (IHY) activities.

[24] Philippa Browning thanks Thomas Woods and another reviewer for their assistance in evaluating this paper.

\section{References}

Bracewell, R. N., and T. W. Straker (1949), The study of solar flares by means of very long radio waves, Mon. Not. R. Astron. Soc., 109, 28-45.
Chilton, C. J. (1961), VLF phase perturbation associated with meteor shower ionization, J. Geophys. Res., 66, 379-383, doi:10.1029/ JZ066i002p00379.

Comarmond, J.-M. (1977), Contribution à l'étude de la basse ionosphère par des mesures de phase et d'amplitude d'ondes électromagnétiques très basse fréquence, Thèse de Doctorat d'Etat, Université Pierre et Marie Curie, Paris VI.

Cummer, S. A. (1997), Lightning and ionospheric remote sensing using VLF/ELF radio atmospherics, PhD Thesis, Stanford Univ., Stanford, Calif.

Hayakawa, M., O. A. Molchanov, T. Ondoh, and E. Kawai (1996), Anomalies in the sub-ionospheric VLF signals for the 1995 Hyogo-ken Nanbu earthquake, J. Phys. Earth, 44, 413-418.

Inan, U. S., T. F. Bell, V. P. Pasko, D. D. Sentman, E. M. Wescott, and W. A. Lyons (1995), VLF signatures of ionospheric disturbances associated with sprites, Geophys. Res. Lett., 22, 3461-3464, doi:10.1029/ 95GL03507.

Kaufmann, P., and M. H. Paes de Barros (1969), Some relationships between solar X-ray bursts and SPA's produced on VLF propagation in the lower ionosphere, Sol. Phys., 9, 478-486, doi:10.1007/BF02391673.

Kaufmann, P., L. R. Piazza, and S. Ananthakrishnan (1973), Possible low ionosphere response to very hard X-rays from Cygnus X-3 bursts in September 1972, Astrophys. Space Sci., 22, 67-70, doi:10.1007/ BF00642822. 
Kaufmann, P., V. L. R. Kuntz, N. M. Paes Leme, L. R. Piazza, and J. W. S. Vilas Boas (1989), Effects of the large June 1975 meteoroid storm on earth's ionosphere, Science, 246, 787-790, doi:10.1126/science. 246.4931 .787$.

Kaufmann, P., L. R. Rizzo Pizza, J. H. Fernandez, and M. Rocha da Silva (2002), Solar flares notproducing sudden phase advances, J. Geophys. Res., 107(A8), 1219, doi:10.1029/2001JA000292.

McRae, W. W., and N. R. Thomson (2000), VLF phase and amplitude: Daytime ionospheric parameters, J. Atmos. Sol. Terr. Phys., 62, 609618, doi:10.1016/S1364-6826(00)00027-4

McRae, W. M., and N. R. Thomson (2004), Solar flare induced ionospheric D-region enhancements from VLF phase and amplitude observations, J. Atmos. Sol. Terr. Phys., 66, 77-87, doi:10.1016/j.jastp.2003.09.009.

Muraoka, Y., H. Murata, and T. Sato (1977), The quantitative relationship between VLF phase deviations and 1-8 $\AA$ solar X-ray fluxes during solar flares, J. Atmos. Terr. Phys., 39, 787-792, doi:10.1016/0021-9169(77) 90140-4.

Nicolet, M., and A. C. Aikin (1960), The formation of the D region of the ionosphere, J. Geophys. Res., 65, 1469-1483, doi:10.1029/ JZ065i005p01469.

Pacini, A. A. (2006), Dependência das propriedades da região-D ionosférica com o ciclo de atividade solar, Dissertação de Mestrado em Geofisica Espacial, INPE, São José dos Campos.

Pacini, A. A., and J.-P. Raulin (2006), Solar X-ray flares and ionospheric sudden phase anomalies relationship: A solar cycle phase dependence, J. Geophys. Res., 111, A09301, doi:10.1029/2006JA011613.

Pant, P. (1993), Relation between VLF phase deviations and solar X-ray fluxes during solar flares, Astrophys. Space Sci., 209, 297-306, doi:10.1007/ BF00627449.

Pant, P., H. S. Mahra, and M. C. Pande (1983), Correlation between observed VLF phase deviation and solar X-ray flux during solar flares, Indian J. Radio Space Phys., 12(2), 40-42.

Pasko, V. P., U. S. Inan, Y. N. Taranenko, and T. F. Bell (1995), Heating, ionization and upward discharges in the mesosphere due to intense quasielectrostatic thundercloud fields, Geophys. Res. Lett., 22, 365-368, doi:10.1029/95GL00008.

Raulin, J.-P., A. A. Pacini, P. Kaufmann, E. Correia, and M. A. G. Martinez (2006), On the detectability of solar X-ray flares using very low frequency sudden phase anomalies, J. Atmos. Sol. Terr. Phys., 68, 1029-1035, doi:10.1016/j.jastp.2005.11.004.

Raulin, J.-P., F. C. P. Bertoni, and H. Rivero (2009a), The South America VLF Network (SAVNET), IAU XXVII General Assembly, Rio de Janeiro, August 2009, Abstract Book, 1, 383-383.

Raulin, J.-P., P. David, R. Hadano, A. C. V. Saraiva, E. Correia, and P. Kaufmann (2009b), The South America VLF NETwork (SAVNET), Earth Moon Planets, 104, 247-261, doi:10.1007/s11038-008-9269-4.

Raulin, J.-P., P. David, R. Hadano, A. C. V. Saraiva, E. Correia, and P. Kaufmann (2009c), The South America VLF Network (SAVNET): Development, installation status, first results, Geofis. Int., 48, 185-193.

Rizzo Piazza, L., P. Kaufmann, and P. Ramirez Pardo (1983), VLF ionosonde and long-distance propagation anomalies produced by galactic Cen X-4 X-ray burst in May 1979, J. Atmos. Terr. Phys., 45, 121 125, doi:10.1016/S0021-9169(83)80016-6.
Rottman, G. J., T. N. Woods, and W. McClintock (2006), SORCE solar UV irradiance results, Adv. Space Res., 37, 201-208, doi:10.1016/j. asr.2005.02.072.

Sato, T. (1975), Sudden fmin enhancements and sudden cosmic noise absorptions associated with solar X-ray flares, J. Geomag. Geoelectr., 27, 95.

Tanaka, Y. T., T. Terasawa, M. Yoshida, T. Horie, and M. Hayakawa (2008), Ionospheric disturbances caused by SGR 1900+14 giant gamma ray flare in 1998: Constraints on the energy spectrum of the flare, J. Geophys. Res., 113, A07307, doi:10.1029/2008JA013119.

Terasawa, T., et al. (2005), Repeated injections of energy in the first $600 \mathrm{~ms}$ of the giant flare of SGR 1806-20, Nature, 434, 1110-1111, doi:10.1038/ nature 03573 .

Thomson, N. R., C. J. Rodger, and M. A. Clilverd (2005), Large solar flares and their ionospheric D region enhancements, J. Geophys. Res., 110(A6), A06306, doi:10.1029/2005JA011008.

Wait, J. R. (1959), Diurnal change of ionospheric heights deduced from phase velocity measurements at VLF, Proc. IRE, 47, 998.

Wait, J. R., and K Spies. (1964), Characteristics of the Earth-ionosphere waveguide for VLF radio waves, NBS Technical Note U.S., 300.

Woods, T. N., W. K. Tobiska, G. J. Rottman, and J. R. Worden (2000), Improved solar Lyman $\alpha$ irradiance modeling from 1947 through 1999 based on UARS observations, J. Geophys. Res., 105(A12), 27,19527,215, doi:10.1029/2000JA000051.

Woods, T. N., F. G. Eparvier, S. M. Bailey, P. C. Chamberlin, J. Lean, G. J. Rottman, S. C. Solomon, W. K. Tobiska, and D. L. Woodraska (2005), The Solar EUV Experiment (SEE): Mission overview and first results, J. Geophys. Res., 110, A01312, doi:10.1029/2004JA010765.

Zigman, V., D. Grubor, and D. Sulic (2007), D-region electron density evaluated from VLF amplitude time delay during X-ray solar flares, J. Atmos. Terr. Phys., 69, 775-792, doi:10.1016/j.jastp.2007.01.012.

F. C. P. Bertoni, E. Correia, H. R. Gavilán, P. Kaufmann, and J.-P. Raulin, Centro de Radio Astronomia e Astrofísica Mackenzie, CRAAM, Universidade Presbiteriana Mackenzie, Rua da Consolação, 896, São Paulo, SP, 01302-907, Brazil. (raulin@craam.mackenzie.br)

P. R. Fagundes, Universidade do Vale do Paraíba (UNIVAP), Av. Shishima Hifumi, 2911, São José dos Campos, SP, 1244-000, Brazil.

G. Fernandez, Complejo Astronómico El Leoncito (CASLEO), Av. España Sur, 1512, J5402DSP, San Juan, Argentina.

W. Guevara-Day, Comisión Nacional de Investigación y Desarrollo Espacial (CONIDA), Calle Felipe Villaran, 1069, San Isidro, Lima, Peru. R. Hadano, Radio Observatório do Itapetinga (ROI) - INPE/Mackenzie, Estrada do Mackenzie, S/N, Bairro Itapetinga, Caixa Postal 200, Atibaia, SP, 12940-000, Brazil

W. L. C. Lima, Universidade Luterana do Brasil (ULBRA), Av. Teotônio Segurado 1501, Plano Dietor Sul, Palmas, TO, 77054-970, Brazil.

A. Pacini, Instituto Nacional de Pesquisas Espaciais (INPE), Av. dos Astronautas 1758, São José dos Campos, SP, 12227-010, Brazil.

R. Rodriguez, Universidad de Piura (UDEP), Av. Ramón Múgica 131, Urb. San Eduardo, Apartado 153, Piura, Peru.

N. J. Schuch and T. R. C. Stekel, Centro Regional Sul-INPE (CRS/ INPE) - Universidade Federal de Santa Maria (UFSM), Caixa Postal 5021, Santa Maria, RS, 97105-970, Brazil. 